\title{
Effects of Swirl Flow on Spray Characteristics in a Swirl-Stabilized Combustor*
}

\author{
Mohammad GHAFFARPOUR** and Alireza NOORPOOR*** \\ ${ }^{* *}$ Department of Mechanical Engineering \\ Faculty of Engineering, University of Tehran, Kargar Shoumali, Tehran, Iran, E-mail: mghaffar@uic.edu \\ ${ }^{* * \star}$ Department of Automotive Engineering, Iran University of Science \& Technology, Tehran, Iran
}

\begin{abstract}
This paper presents experimental data on the characteristics of a kerosene spray flame produced by a hollow-cone pressure-atomized nozzle in a swirl-stabilized combustor. These acquired data include the volume flux distribution, size and velocity distribution, measured by use of Phase Doppler Particle Analyzer (PDPA). The effects of swirl number on different parameters, recirculation zone, and stability of the flame is investigated. The data is offered for use in assessments of two-phase flow computational methods as are useful to gas turbine and furnace combustors design procedure. The overall characteristics of this spray flame were defined.
\end{abstract}

Key words: Spray, Flame, Swirl, Phase Doppler Particle Analyzer

\section{Introduction}

In two-phase reacting designing more fuel-efficient and less environmentally destructive power generation/conversion devices is a major challenge. Gas turbine engines, liquid rockets, furnaces, incinerators, and diesel engines are typical examples in which spray combustion is of prime interest. The performance of liquid fueled reacting system is due to formation, evaporation and burning of liquid fuel droplets. The atomization and evaporation of liquid fuel, the mixing of the evaporated fuel with the swirl and dilution air, and the burning of the resulting two-phase mixture are the most important parameters to evaluate the combustion efficiency, stability, and emission.

Labs and Parker ${ }^{(1)}$ measured droplet diameter and volume fraction for three different fuels (diesel fuel, dodecane, and methyl oleate) as a function of radial position within a pressure-atomized. The measurements were carried out into atmospheric pressure and ambient-temperature air at an axial distance of $25 \mathrm{~mm}$ from the nozzle. The results indicated droplet diameters are the smallest at the spray centerline, and grow with radial distance from the centerline. Oh et al., ${ }^{(2)}$ investigated the kerosene spray and the spray flame structure using an air-assisted atomizer. A particle dynamic analyzer (PDA), a thermocouple and direct photographic technique were used. Three representative flames were studied and measurements are made at three different axial locations from the nozzle, with and without combustion. Detailed comparison of the spray structures in nonburning and burning conditions was presented. Results indicated that the overall structure of an air-assisted spray flame is different from that of gaseous diffusion flames and is characteristically similar to internal group combustion mode where liquid and gas phase reactions coexist.

Widmann et al., ${ }^{(3)}$ used Phase Doppler interferometry (PDI) measurements in low number density sprays. They investigated the effect of insufficient sample statistics on the calculated probe area, and the resultant uncertainty in the volume flux measurement. Widmann et al., ${ }^{(4)}$ also showed that burst splitting has a serious effect on volume flux measurements; however, the impact on size and velocity distributions is much weaker. The events significantly distort the probe volume correction, which is applied to the measured size distributions to remove the bias introduced by the size dependence of the probe area.

Practical limitations associated with the use of small probe volumes with respect to the droplet size that is being measured by the phase-Doppler interferometry technique were 
discussed by Strakey et. al., ${ }^{(5)}$ Volume flux measurements in dilute sprays showed a significant improvement over those made by standard phase-Doppler interferometry techniques at small beam waist/droplet size ratios. Gupta et al., ${ }^{(6)}$ obtained data on droplet size, number density, and velocity at several spatial locations in the spray flames using a two-component phase Doppler interferometer in a twin-fluid atomizer. They concluded that global features of the spray flames were affected dramatically by oxygen enrichment of the atomization.

Saumweber et al., ${ }^{(7)}$ used new method in a two-phase flow produced within an annular air jet has resulted in profiles of axial mean and fluctuating velocities and shear stresses as well as integral time scales and turbulent energy spectra. It was shown that the spray with a very small quantity of dye preferentially attenuates the light intensity scattered by large droplets, thus allowing the PDI system to detect small seed particles without damaging the photodetectors when large particles are present in the flow. Bulzan ${ }^{(8)}$ measured drop size and mean velocity and drop number flux for five axial distances downstream in a reacting swirl-stabilized combustor using a two-component phase/Doppler particle analyzer. Mean velocity measurements for the gas phase were reported for both isothermal cases.

The main goal of any swirl-stabilized combustion system is to achieve higher efficiency and performance, and lower emissions, which is directly related to the flame stability. This is encouraged by the use of a recirculation zone, which causes the combustion products to push back towards the center of the flame and combustion chamber. Therefore, it acts as an ignition source and keeps the flame stable. A recirculation zone also aids in the burning of unspent fuel.

A recirculation zone can be obtained with any one of three ways: bluff body, sudden expansion, or swirl. A bluff body is a good demonstration of the Bernoulli principle. By simply a non streamlined object placed into an oncoming stream, the bluff body causes the fluid to move around it. Recirculation by sudden expansion is when a fluid is moving through a pipe that suddenly increases in diameter by a large magnitude.

The third way of achieving a recirculation zone, which is used in this experiment, is by employing a swirl plate. A series of vents radially aligned and at a fixed angle to the central axis, cause the fluid to flow outwards in a spiraling motion. The centrifugal force associated with the angular momentum causes a low pressure zone inside the vortex of the swirl. This low pressure zone draws in the fluid. The most common way of quantifying the characteristics of swirl flow is with the swirl number. The swirl number is a dimensionless parameter, defined as the angular momentum flux divided by the axial momentum flux. Therefore for a given flow rate of air, the lower the angle of the fins on the swirl plate with respect to the chamber axis, the more the air is directed axially, hence the lower the swirl number.

The combustion characteristics of gaseous diffusion flames are controlled mainly by the chemical kinetics and mixing between fuel and air. In two-phase reacting flow, evaporation rate, droplet size and velocity depend on the condition at the fuel nozzle and the interaction between the sprays and the surrounding air and can control the combustion rate. It has been shown that droplets control the combustion process. The flow field in a Gas Turbine Combustion (GTC) and furnace chambers are three-dimensional and complex with interaction between primary jet, dilution jet, swirl air, wall cooling air, and fuel flows. Accurate and extensive measurements of volume flux, drop velocity, drop velocity fluctuation, and drop size are extremely difficult in a real combustor since the accessibility is difficult and having harsh environment.

Note that there are two mixing processes of importance in the primary zone of conventional combustor: One involves the mixing of fuel vapor with hot burned gas in the recirculation zone and the other that of the resulting fuel-burned gas mixture mainly with the swirling air. Therefore, to investigate the fluid-mechanical characteristics of a hollow-cone spray flames and the effects of the swirl number on flame stability similar to those occurring in the primary zone of gas turbine and in the furnace combustion chambers, a swirl-stabilized combustor was used to simulate a part of the events occurring in an actual GTC and furnace chamber. The objective of this work is to investigate effects of some parameters of PDPA such as PM tube voltage and velocity shift on volume flux; investigating the effects of swirl number on the recirculation zone and the flame stability; 
and offering some results for modeling and computational efforts.

\section{Experimental Setup and Instrumentation}

Fig. 1 shows a schematic drawing of the combustor. The combustor consists of a circular cross section stainless steel pipe of $265 \mathrm{~mm}$ in diameter and $768 \mathrm{~mm}$ in total length that is positioned in a vertical direction. An internal pipe of $98 \mathrm{~mm}$ in diameter and $254 \mathrm{~mm}$ in length, through which swirl airflows, is installed at the lower end of (and concentric with) the outer pipe. The main air from the compressor goes through a regulator/filter assembly and is divided into two branches, one for dilution air and the other for swirl air. Air in each branch passes through a regulator, a valve, and a rotameter to a manifold from which four pipes are drawn to the lower end plate of the combustor for uniform air distribution. Each pipe ends in a specially designed distributor to spread the air evenly in all directions. A distance of about $102 \mathrm{~mm}$ from the end plate to the lower face of the honey comb was found to be sufficient to allow proper and uniform air distribution inside the combustor. Fuel from the tank passes through filters and is pumped by a fuel pump into an on-axis vertically adjustable tube after passing through a rotameter for fuel flow rate measurements.

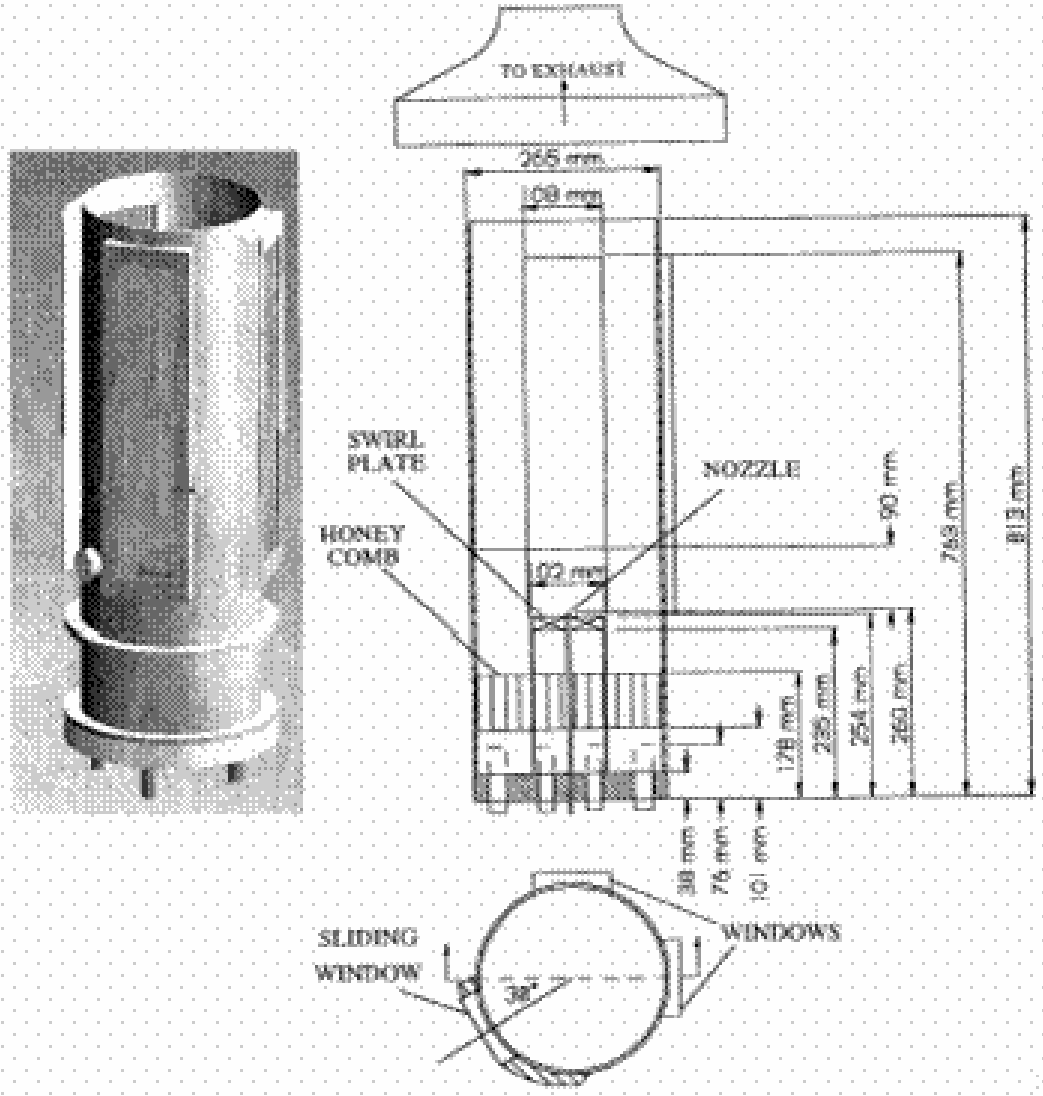

Fig. 1. General view and schematic drawing of the Combustor

Bell Super K-1 kerosene is used for all the experiments and was ignited via a small sidewall opening with a propane torch, which is subsequently removed. An industrial type pressure-swirl nozzle, producing a hollow-cone spray with nominal total spray angle of $30^{\circ}$ and rated flow rate of $38.5 \pm 0.99 \mathrm{cc} / \mathrm{min}$ (fuel density of $0.8 \mathrm{gr} / \mathrm{cc}$ ) and constant injection pressure of 7 atmosphere, is used to produce an axisymmetric spray at the end of the fuel tube in the combustor. A swirl plate is placed just under the nozzle, which has a series of vents that generate a clockwise recirculation zone. In this experiment, two different swirl plates were used. In one plate, the vents were set at an angle 30 degrees to the axis of the chamber. The other swirl plate was set at 45 degrees. The changing of the vents' angle was found to affect the flow field in the combustion chamber. The combination of the flow and the vents 
being along a helical path produces a toroidal shaped flow field. The air swirl plate (Fig. 2 shown for 30 degrees swirl plate) is installed flush with the upper edge of the swirl pipe.

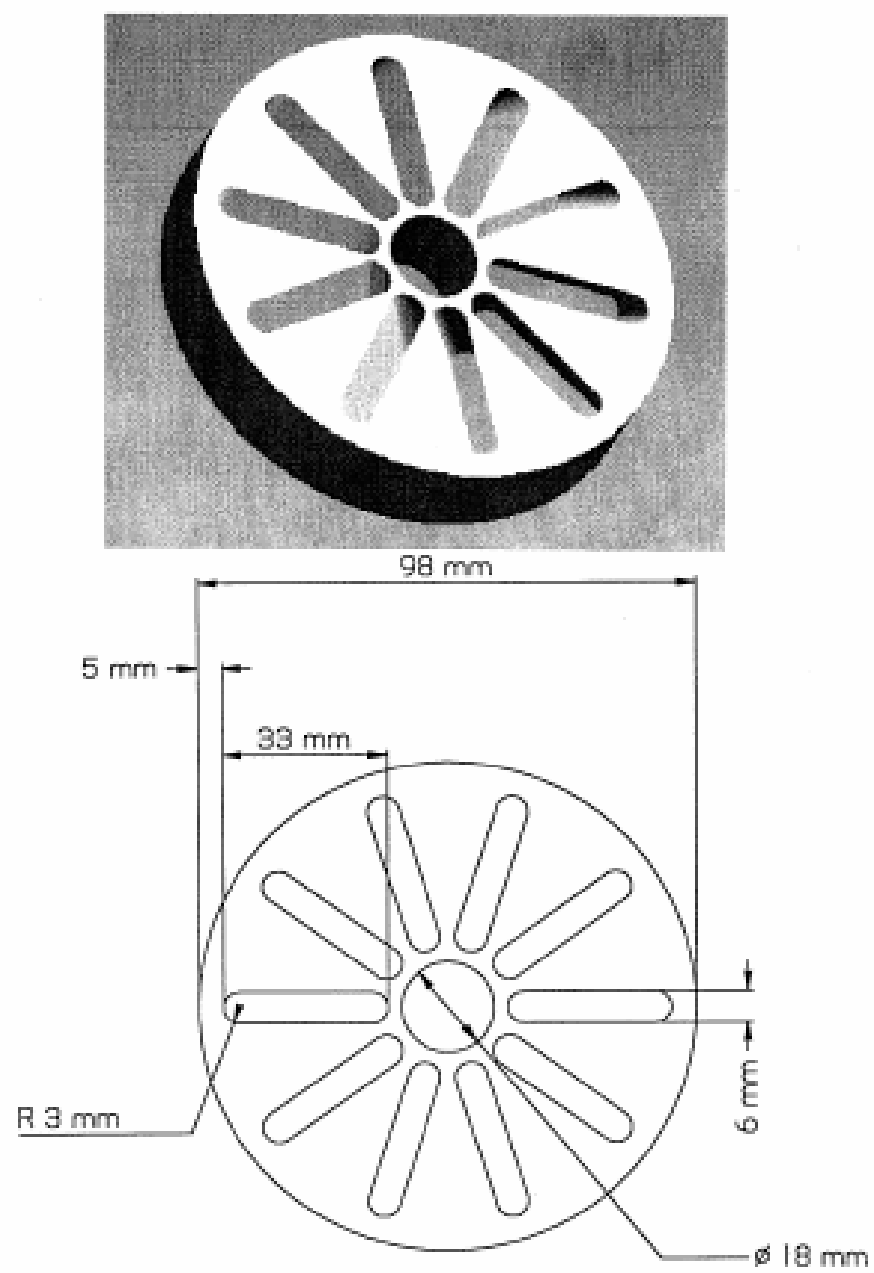

Fig. 2. General View and schematic drawing of the swirl plate

Looking into the swirl plate one sees ten radial slots, $33 \mathrm{~mm}$ in length and $6 \mathrm{~mm}$ in width equally spaced tangentially, which generate clockwise swirl flow. These slots generate a calculated swirl number of about 1.5 (for 30 degrees swirl plate) and 2.55 (for 45 degrees swirl plate) with the design exit velocity at an angle of $30^{\circ}$ and $45^{\circ}$ with respect to the chamber axis (9). Three $514 \mathrm{~mm} \mathrm{X} 108 \mathrm{~mm}$ semi-conductor grade high temperature fused quartz windows are installed for optical access into the combustor. Two of the windows are at $90^{\circ}$ with each other, with the third being at $150^{\circ}$ from one of them. This is required due to optimized operating arrangement of our laser instrumentation. These windows are kept warm enough during the experiment to remove soot. Also, after every 3-4 hours of the experiment, the windows are cleaned by heating and cleaning the windows by gasoline.

A single-component Phase Doppler Particle Analyzer (PDPA) by Aerometrics, model No. XMT-1100 (Optical Transmitter), RCV 2100 (Optical Receiver) was used to simultaneously measure drop size and velocity. Both receiving and transmitting optics were rigidly mounted on a three-dimensional traversing table with one digital readout for each direction. Receiving and transmitting lenses were $495 \mathrm{~mm}$ f.l. (i.e. focal length) with f/no. 4.7 and 495 f.l. with f/no. 7 respectively. A colliminating lens with 300 f.l. and a slit width of 100 $\mu \mathrm{m}$ were used in the receiving optics. Note that the PDPA comes with a certain set of transmitting and collimating lenses from which the above-mentioned arrangement was selected as being optimum considering the geometrical dimension of the chamber and tested condition. The beam waist diameter with this arrangement was $176 \mu \mathrm{m}$. 
PDPA theory and sizing accuracy has been fully described in many references such as Bachalo ${ }^{(10)}$, Bachalo and Houser ${ }^{(11)}$, Sankar and Bachelo ${ }^{(12)}$, and Sankar et al. ${ }^{(13)}$. For accurate determination of the mean and median droplet sizes, along with accurate measurements of volume flux and number density, all droplet size classes must have an equal probability of detection. Without correction, the PDPA exhibits sample volume bias due to the Gaussian intensity profile of the laser beams. The PDPA software performs a sample volume correction based upon an empirical determination of the maximum sample volume size for each size class in order to give all size classes equal probability of detection. This correction is imperative for accurate flux and number density determination, which can be within $\pm 10 \%$ with proper high voltage setting ${ }^{(14)}$. PMT gain was set via the intensity function.

\section{Results and Discussions}

For a set of fixed fuel atomizer parameters and a given swirl plate design there are basically two main inputs, dilution and swirl airflows. To characterize the stable operating conditions of the combustor, the behavior of the flame was studied by photography and video camera. The most stable flame was found to exist around 3.69/1.55 for 30 degrees swirl plate (a swirl flow rate of $3.69 \mathrm{~kg} / \mathrm{min}$ and dilution flow rate of 1.55 $\mathrm{kg} / \mathrm{min}$ ). Note that all experimental results were taken at 3.69/1.55 conditions.

The color photos and video images (not shown here) at the most stable condition showed several regions of the spray flame. However, the converted color photograph is shown in Fig. 3. Very close to the nozzle, a conical liquid fuel sheet existed that disintegrated into droplets. This region was up to about $10 \mathrm{~mm}$ above the nozzle. From $10 \mathrm{~mm}$ to about $20 \mathrm{~mm}$ the time-averaged color photos for $3.69 / 1.55$ conditions showed exclusively light-blue region surrounded by spray droplets made visible by laser light. This color is due to slower evaporation rates of droplets and very lean mixture. At the same condition from $20 \mathrm{~mm}$ to $35 \mathrm{~mm}$ from fuel atomizer, a mixture of blue and yellow colors was seen. Exclusively yellowish flame due to soot was detected above $35 \mathrm{~mm}$ from the fuel atomizer.

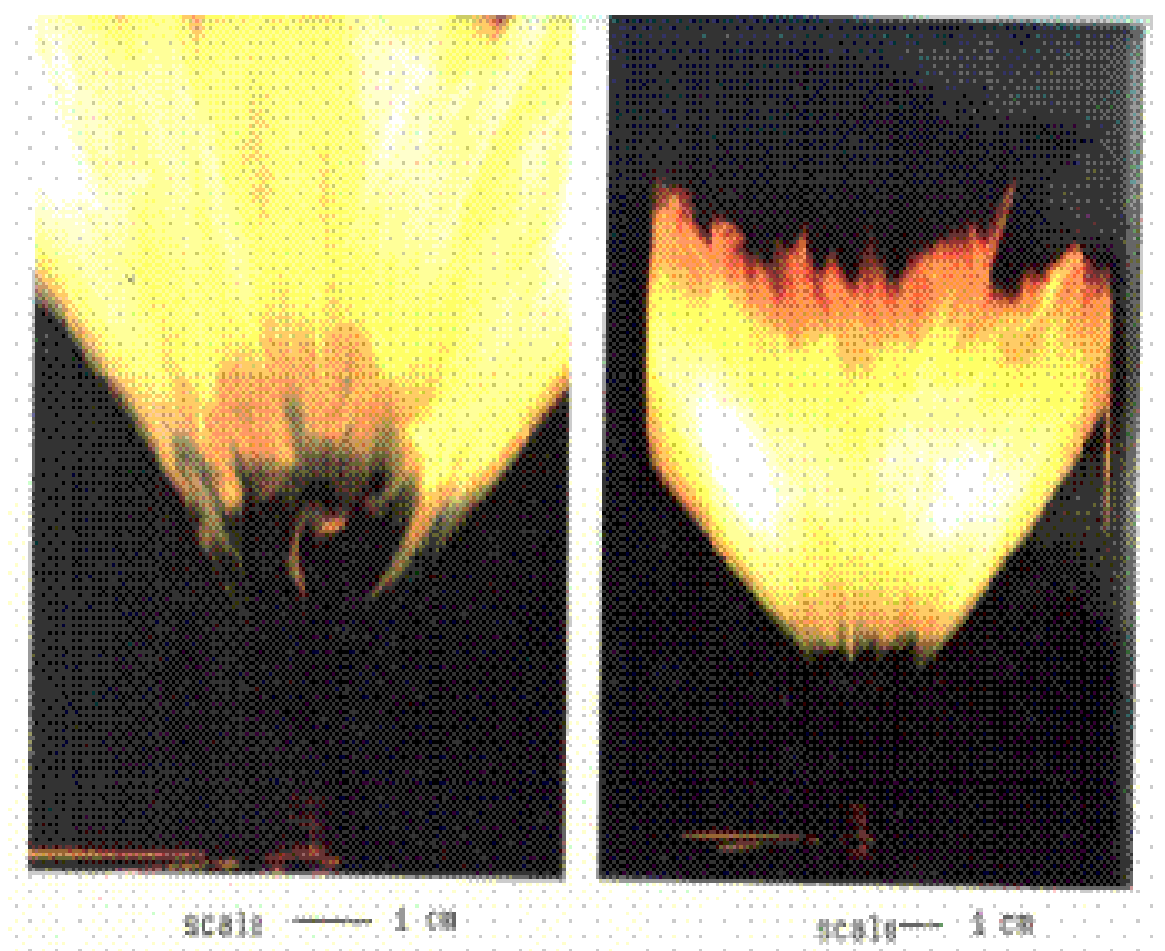

Fig. 3. Color photograph of the flame at 3.69/1.55 condition to black and white

Bachalo et al. (15), Chehroudi (16), and McDonell and Samuelsen (17) showed changes in some of the results obtained by a PDPA in sprays if "optimum and/or proper" 
choices of these instrument variables (PM-tube voltage and shift velocity) were not made, particularly in the dense regions of sprays. Therefore, prior to measure the fuel volume flux and (mass) flow rate and percentage of fuel evaporation at different axial locations; to investigate the mixing and diffusion rates into the flame and the other parameters; and offering some explanation for the profile of the volumetric flow rate and its accuracy, it is necessary to discuss the variation of the volume flux with the PM tube voltage and the velocity shift since values of the volumetric flow rate highly depends on these parameters. So, two representive points, one within the flame $(\mathrm{r}=3.75 \mathrm{~mm}$, up triangle symbol (solid for combustion and hollow for no combustion) in Figs. 7, 8, 9) and one close to the spray axis $(\mathrm{r}=1.75 \mathrm{~mm}$, down triangle (solid for combustion and hollow for no combustion) in Figs. 7 , $8,9)$ were chosen at an axial position of $15 \mathrm{~mm}$ to investigate the effects of these instrument parameters on volume flux.

Since the intensity of the scattered light is proportional to the square of drop size, only a specific range of voltage for a given size distribution can be used. Effects of photomultiplier (PM) tube voltage on volume flux are shown in Fig.4 for the cases with (solid symbol/solid line) and without (hollow symbol/dotted line) combustion. Lowest to highest PM voltages correspond to unpractical data acquisition time and PM tube saturation respectively. For voltages below and at 250, the volume flux is too low due to undetected droplets for this spray. As the voltage increases, photodetectors become more sensitive to the scattered light which may cause a small droplet to be sized as larger droplet than the set limit by the operator and consequently will be rejected. Fig. 5 shows that an increase in the PM tube voltage increases the sampling area, leading to larger droplet residence times and increased overflows. Increasing the voltage also increases the noise level, which affects the signal that is critical to the measurement of the phase shift. Therefore, it increases the error in the phase shift value leading to higher bad-data rejection percentage and consequently reduction of the measured volume flux for high voltages. The manufacturer does not suggest a very low or high PM tube voltages (250 and 450) in this spray. For the purpose of this paper, the shapes and the order of magnitude of the plots are used later in volume flux measurement discussions.

From Fig. 4, two measurement procedures can be considered: adjust the gain on the PM tube to the local plateau value at each measurement location and condition or choose a constant compromise value for all locations. The latter approach is more practical and was adopted for convenience. Note that the choice of this voltage is not unique and usually a range of voltage exists for optimum operation. No plateau exists in the volume flux profile and a value of $350 \mathrm{VDC}$ seems to be a good compromise.

Fig. 6 presents effects of frequency (or velocity) shifting achieved by rotating diffraction grating on volume flux. The manufacturer of the PDPA does not encourage large values of the shift velocity (or frequency) in the operating manual. For a given voltage a slight change in volume flux has been observed while increasing the velocity shift for the case with combustion. Calculations showed that $20 \mathrm{~m} / \mathrm{s}$ shift velocity were sufficient to minimize the acceptance-angle effect (i.e. fringe bias) in our situation and especially with combustion $^{(16)}$. A $20 \mathrm{~m} / \mathrm{s}$ shift velocity was chosen for all the results presented hereafter. Effects of PM tube voltage and velocity shift on volume flux at axial position of $40 \mathrm{~mm}$ also gave similar results and conclusions.

Fig. 7 shows the mean droplet velocity at different axial positions of 15-90 mm from the nozzle, as well as at different radial positions starting at the central axis of the chamber and ending at the inner diameter of the chamber. On the right side the symbols report their velocity for the case of combustion, and on the left they represent a no-combustion case, all for the 30 degree swirl plate. On each side there are also lines, rather than symbols, which reflect the same data but when a 45 degree swirl plate is used. Also present in the Fig., are horizontal lines which symbolize zero velocity. For a given radial position, the droplets above the line have a positive upwards velocity, and any droplets below that line are moving downwards. Rejection percent for these data is at most 20 and beam steering was visually observed not to be significant, in agreement with McDonnell and Samuelsen $\left({ }^{17}\right.$. The two inner upgoing-diverging lines represent the extent of spray if it penetrated at nominal spray initial angle. 

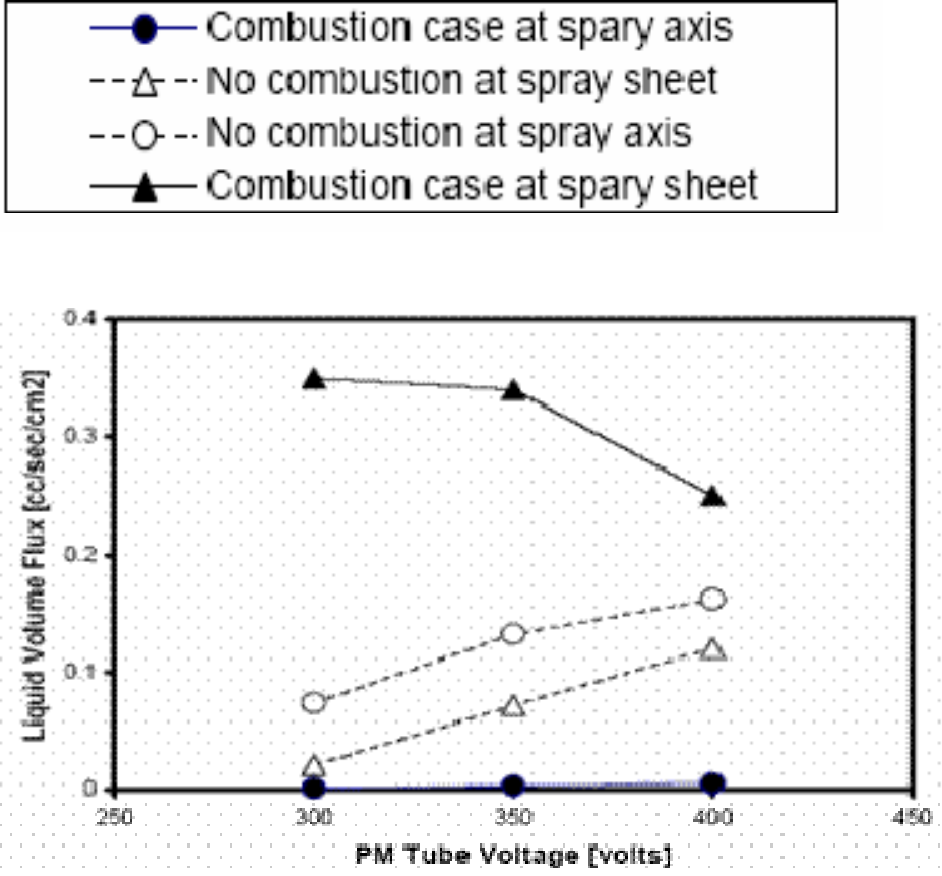

Fig. 4. Effect of the PM-tube voltage on measured volume flux

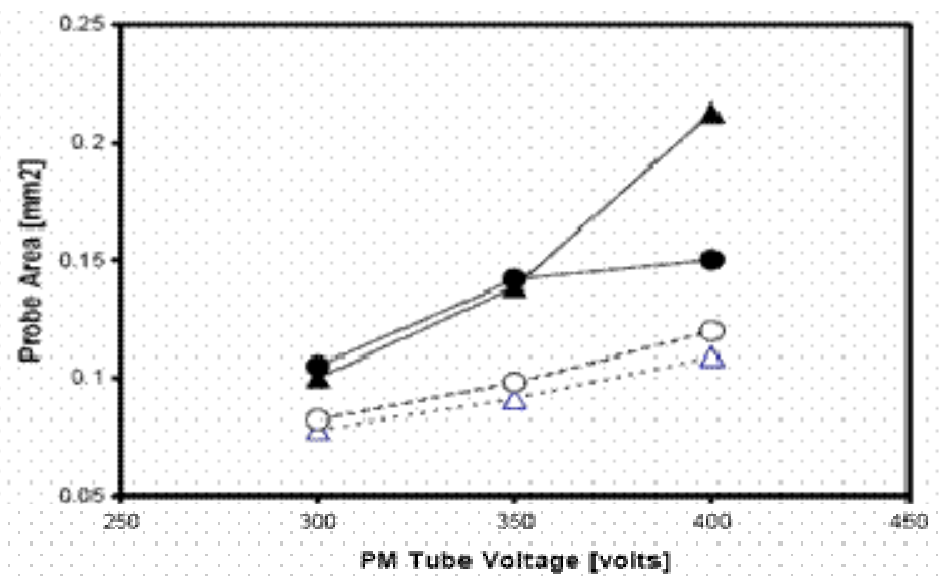

Fig. 5. Effect of the PM-tube voltage on measured probe area

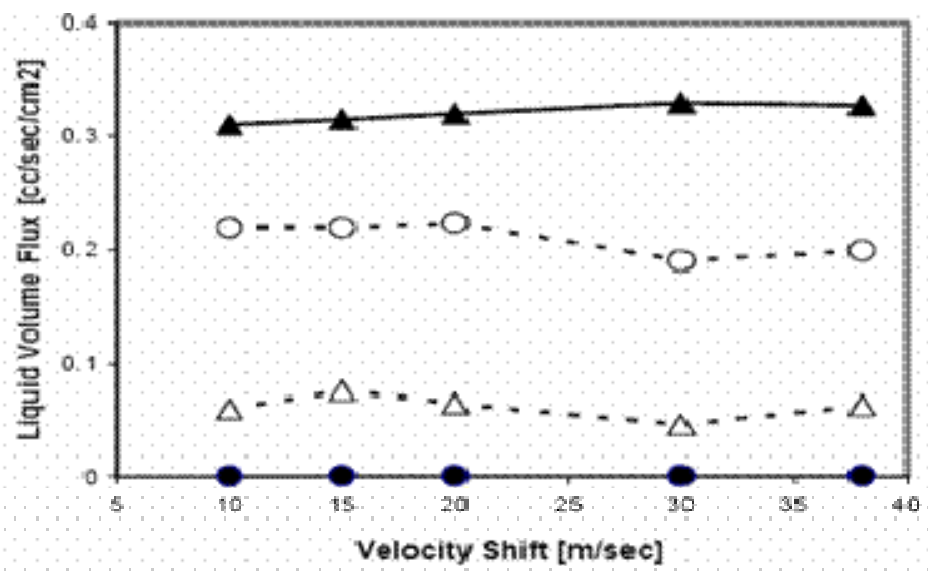

Fig. 6. Effect of velocity shift on measured volume flux 
Mean drop velocity profiles for the non-burning case (for 30 degrees swirl plate) show its minimum at the center and its maximum away from the center of the chamber. Negative mean drop velocities are seen to exist around the spray center line for axial locations of above $25 \mathrm{~mm}$ for 30 degrees and above $40 \mathrm{~mm}$ for 45 degrees swirl plate, indicating the existence of a toroidal recirculation zone.

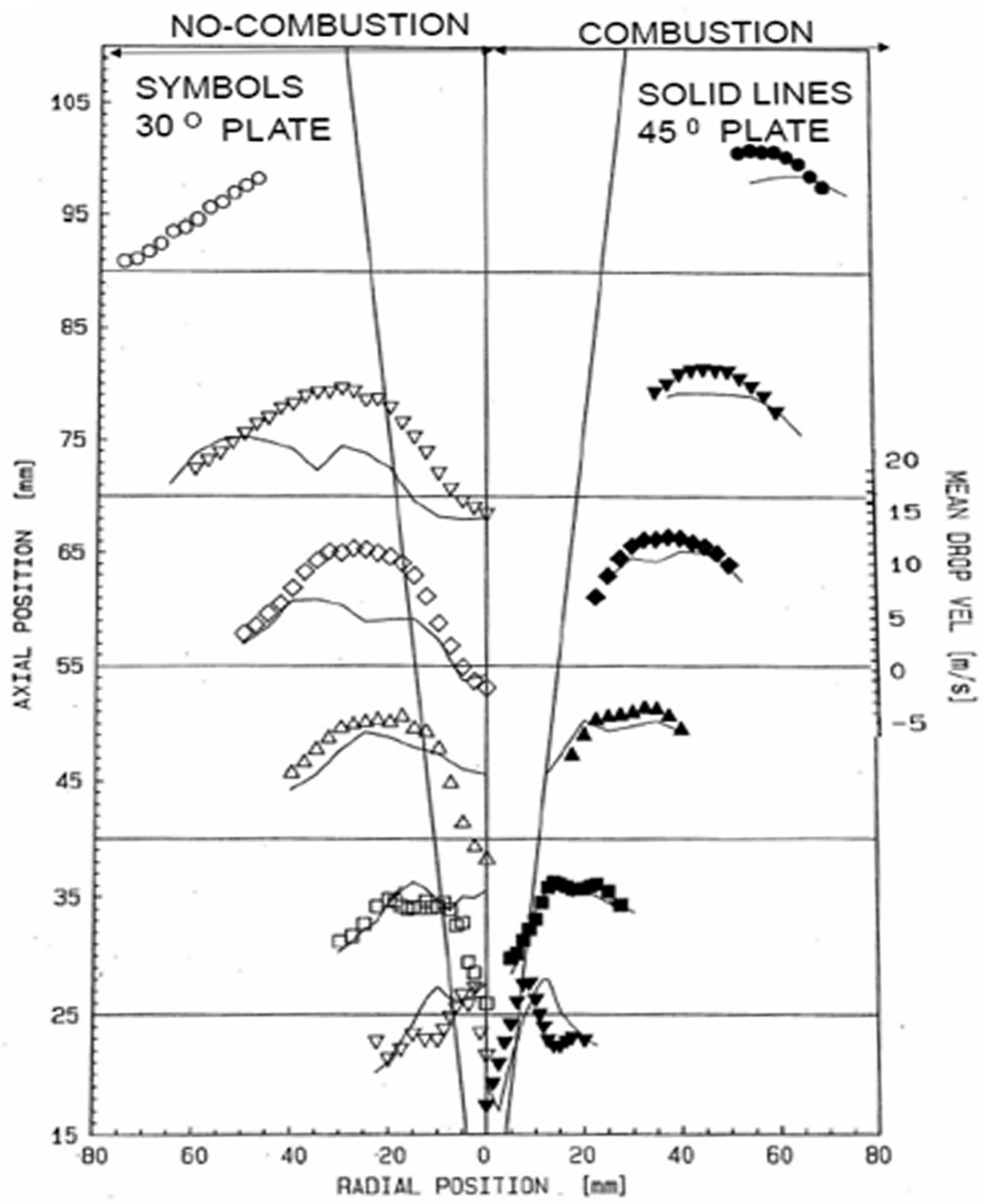

Fig. 7. Offset plots of the mean axial drop velocity for combustion cases (right side) and for no-combustion case (left side) at six different axial locations. Symbols for 30 degrees swirl plate and solid lines for the 45 degrees

For the 45 degrees swirl plate, mean drop velocity profiles show a double-hump shape. 
Negative mean drop velocities are around the spray center line for axial locations of above 55 $\mathrm{mm}$, indicating the existence of a toroidal recirculation zone, which is shifted to the top of the chamber from $25 \mathrm{~mm}$ to $55 \mathrm{~mm}$ from the nozzle as is compared with 30 degrees swirl plate. Generally, the mean drop velocity is higher for 30 degrees swirl except close to the nozzle since the jet flow angle is lower.

In the measurements with combustion, no drops were found in the central region above the $15 \mathrm{~mm}$ axial position for two swirl plates. Radial expansion of the spray is evident by the outward shift of the peak drop velocity profiles with combustion. Also if velocity profiles with and without combustion for each cases at any fixed axial location are horizontally shifted to align the peaks along a vertical line, one can then observe higher mean drop axial velocity with combustion. Note that experiments for both cases of swirl plates with and without combustion were performed in the same combustor setup with identical swirl and dilution air flow rates and so effects of spray combustion were isolated. At a $15 \mathrm{~mm}$ axial distance, which is just within the blue flame region (on the average), the mean drop axial velocity for the combusting case is considerably lower than for the isothermal case close to the spray axis. This is perhaps due to the downward movement of the recirculation zone. At $15 \mathrm{~mm}$, the peak of the mean drop velocity profile for the nonburning case is very close to the nominal spray angle, which later departs from it away from the nozzle. This departure is due to the swirling air and turbulent diffusion. The 45 degree swirl plate creates some interesting differences. For the combustion case, the drops never achieve as high of a peak velocity as 30 degree swirl plate, and within a certain range ( 40 to $70 \mathrm{~mm}$ from nozzle) in the chamber, they form a double hump pattern.

Fig. 8 shows plots of RMS drop velocity fluctuations (for 30 degree swirl) using the same format as in Fig. 7. For the nonburning case, the profiles show the typical double hump for almost all axial position, it also show a local minimum at the center line with peaks at about the inflection points of the corresponding mean drop velocity profiles in Fig. 7. In this case, the absolute minimum velocity fluctuation occurs almost at the spray sheet for all axial positions except those that are close to nozzle $(Z=15 \mathrm{~mm}$ and $Z=25 \mathrm{~mm})$. This may be due to the beginning of the spray sheet break up as was observed by photography. The only appreciable difference between results with and without combustion occurs in the outer region of spray. However, if the profiles are horizontally shifted such that the peaks of their corresponding mean velocity profiles align, then the rms of drop velocity fluctuations for the burning case will always be lower than the isothermal ones. This lower value for the combusting spray is perhaps due to evaporation of small drops (see Fig. 9) and increase in gas kinematics viscosity at high temperatures. Comparing the two swirl plates, it can be seen that the axial positions closest to the nozzle with the 30 degree swirl plate exhibit the largest RMS. In axial positions further away from the nozzle, in the case of combustion, the RMS tends to flatten out, with only a slightly increased RMS on the inside and outside extremes. The 45 degree swirl plate with combustion has very similar behavior, but the RMS is higher. Seeing as how turbulence was predicted for the 45 degree swirl plate in this analysis, it seems likely that this turbulence would cause a higher RMS of velocity fluctuation. However, for the non-combustion case, the opposite can be seen. For the nonburning cases and 30 degree swirl plate, the RMS of drop velocity fluctuates near the inside of the chamber (within spray sheet). At the outer region of spray sheet, it takes a more flattened form. This shape is fairly consistent among the different axial positions. The shape for the 45 degree plate for nonburning case changes considerably as the spray advances upwards. For the first two axial positions, the peak of RMS of velocity fluctuation is well outside of the spray cone angle. At the $40 \mathrm{~mm}$ axial position, the peak shifts to the center of the chamber. At axial position of $15 \mathrm{~mm}$ from nozzle, the peak shifts over to the spray cone, and it is the lowest at the center of the chamber. It can be concluded that this drastic change is a good indication that the 45 degree plate is not favorable for a stable flame.

Fig. 9 shows the SMD profiles with and without combustion. Horizontal line at each axial position represents base SMD level of 20 microns. SMD radial profiles with combustion show increases in almost all locations due to preferential evaporation of small drops $\left(\mathrm{d}^{2}-\mathrm{Law}\right)$ that shift the probability distribution function of drop sizes towards the larger drops. For swirl plate of 30 degrees and the isothermal case, they portray the classical double-hump profile with large drops close to the spray nominal spray angle surrounded by deflected small drops. Larger droplets have more initial momentum, which can penetrate farther and to wider distance. 
For the 45 degree swirl plates, due to higher turbulence, there is a rather strange distribution of drop diameters that don't seem to follow any trends. For the 30 degree swirl plate in the non-combustion case, it can be seen that the radial position of the maximum SMD for each axial position follows along the initial cone-angle for the nozzle. This proves that since larger drops have greater mass and are less likely to deviate from their

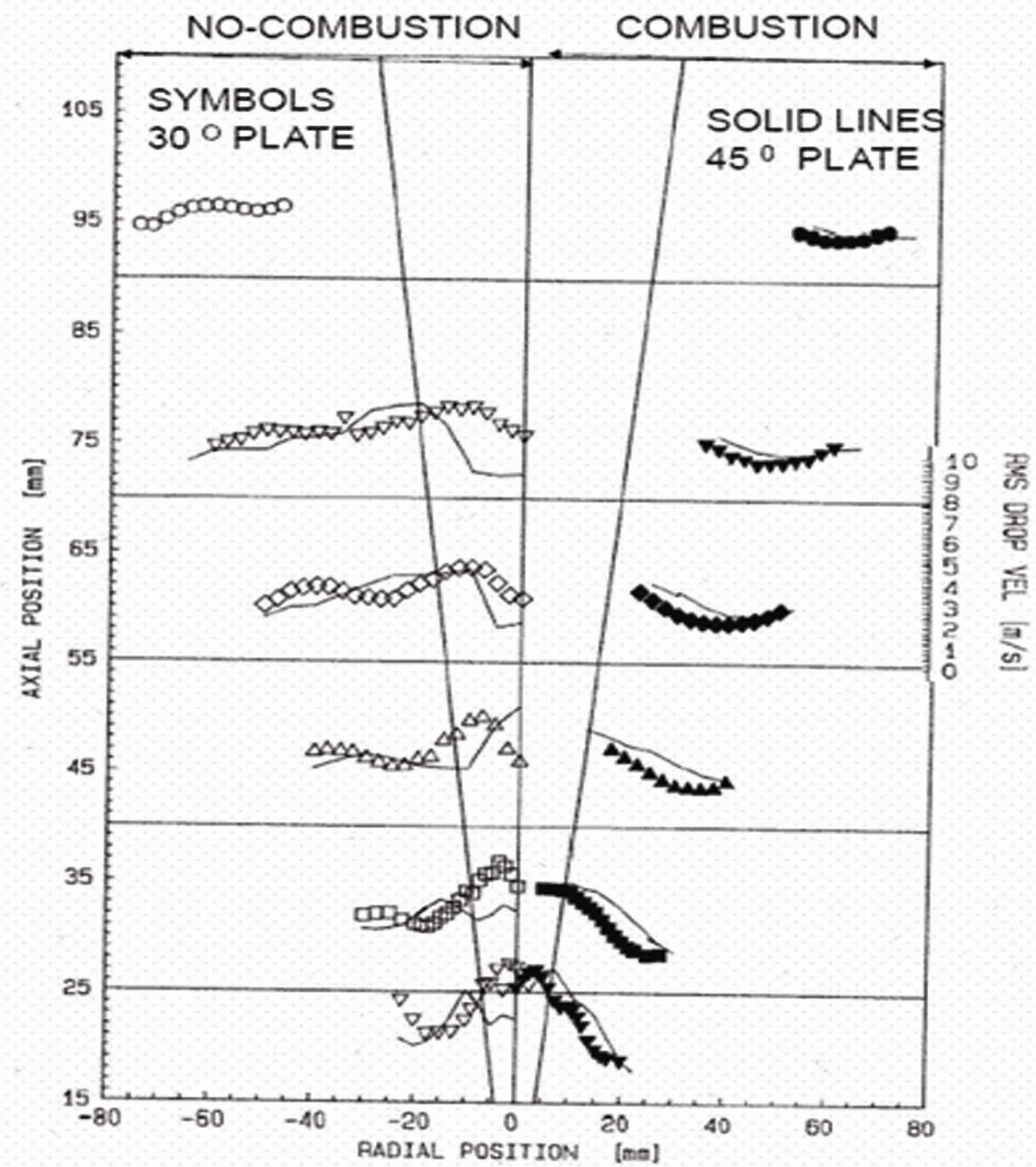

Fig. 8. Offset plots of the velocity fluctuations for combustion cases (right side) and for no-combustion case (left side) at six different axial locations. Symbols for 30 degrees swirl plate and solid lines for the 45 degrees initial path, the largest SMD is found along this initial cone-angle. The combustion case is slightly more complicated. For the first couple axial positions, the plot shows a very similar SMD distribution to the non-combustion case. However as the fuel passes through the chamber, it gets consumed by the flame. The flame appears to be in a conical shape, following the same basic shape as the fuel leaves the nozzle. This shape becomes more distorted at far axial distances from the nozzle. Between the axial positions 40 and $70 \mathrm{~mm}$, just outside of the conical flame region, are the larger droplets. This agrees with the fact that the heaviest drops will follow their initial trajectory. But according to the plot, the drops 


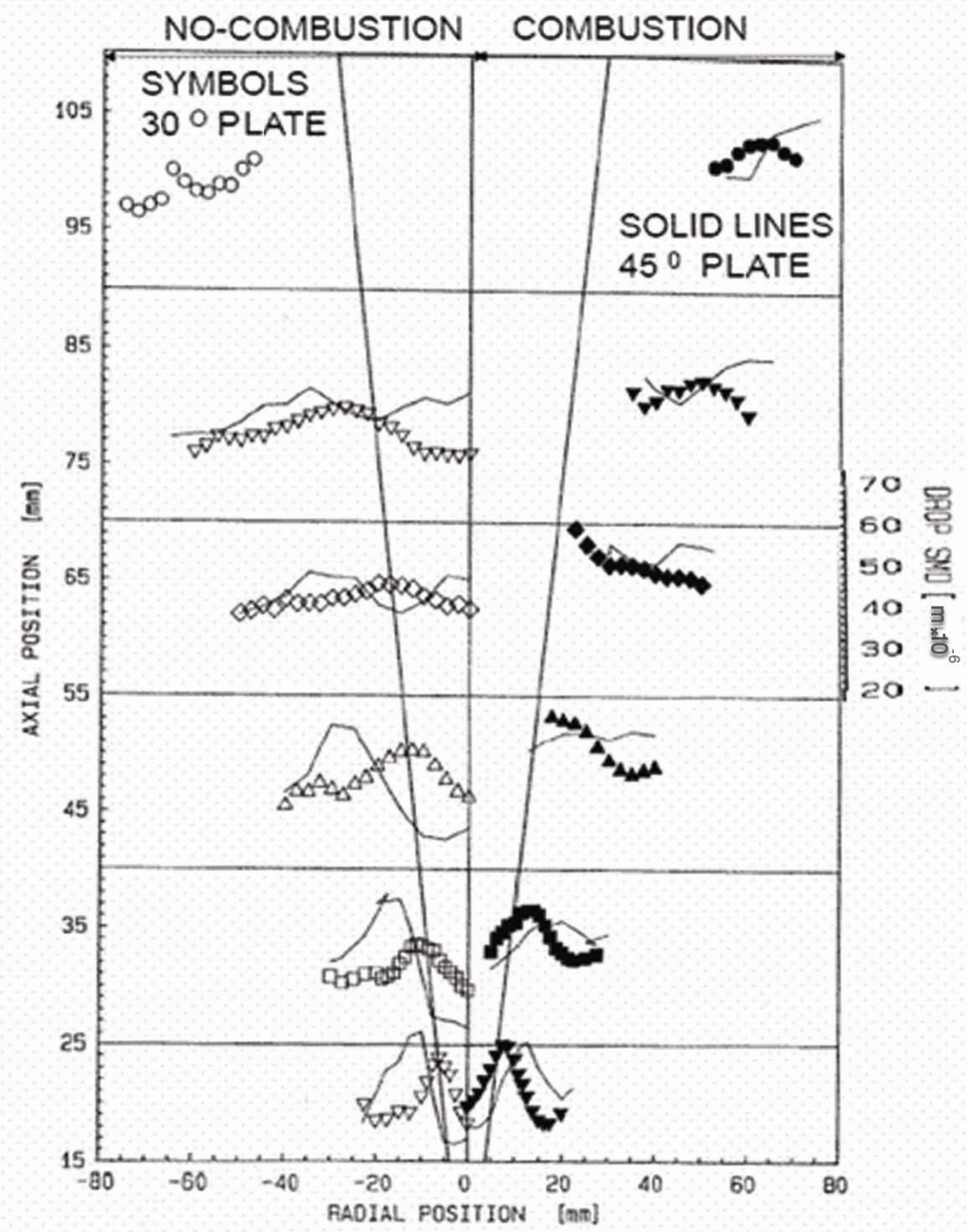

Fig. 9. Offset plot of the SMD for combustion cases (right side) and for no-combustion case (left side) at six different axial locations. Symbols for 30 degrees swirl plate and solid lines for the 45 degrees

furthest away from the flame have the lowest SMD (not the case for the 45 degree swirl plate). This means that the smaller droplets are straying away from the initial spray angle, and that behavior is also concurrent with the non-combustion case. Then as the droplets go beyond the $75 \mathrm{~mm}$ axial position, the trend shifts to where the lowest SMD can be found closest to and furthest from the flame. This is likely due to evaporation.

Fig. 10 shows the radial liquid volume flux profiles with and without combustion at six different axial locations. The format of this plot is the same as the previous plots. Note that the scale for the lowest three axial positions is one-tenth of that for the upper three plots.

The liquid volume flux is one parameter, which characterizes and evaluates the evaporation and mixing rate. For swirl plate of 30 degrees and the non-burning case, the volume flux increases with radial distance to a maximum value and then decreases toward the outer edge of the spray. The peaks of the liquid flux at downstream axial positions are lower. 


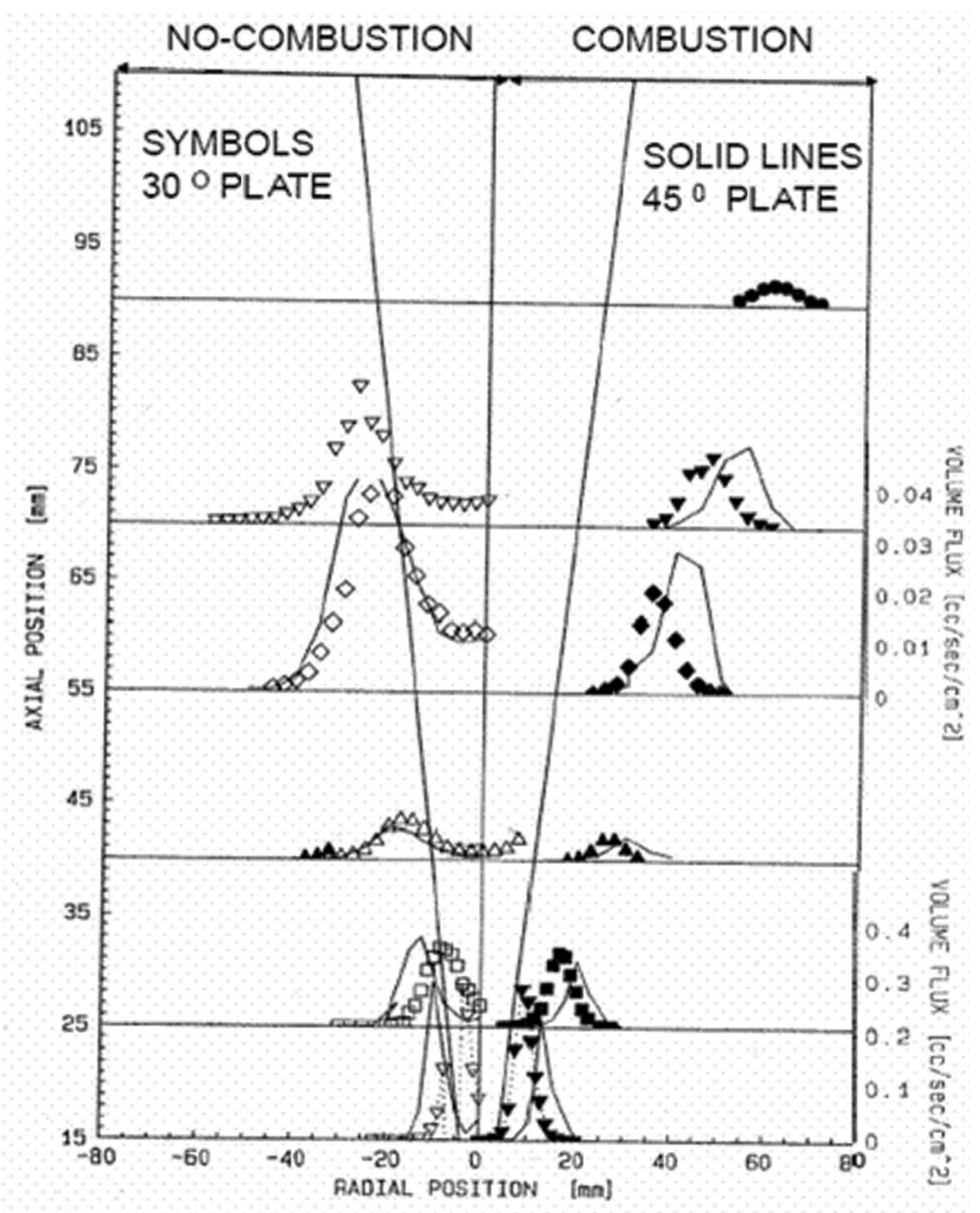

Fig. 10. Offset plots of the liquid volume flux for combustion cases (right side) and for no-combustion case (left side) at six different axial locations. Symbols for 30 degrees swirl plate and solid lines for the 45 degrees

In this case the peak of the volume flux for the non-burning case occurs close to the theoretical initial spray angle and decays in magnitude downstream of the atomizer. Minimum volume flux is shown to be at the spray centerline. Note that the volume flux is determined from the measured volume mean diameter $\mathrm{D}_{30}$, sampling cross section area, and the number of particles counted in the total sampling time. The sample volume is corrected by the PDPA software for the effects of the flow angle ${ }^{(15)}$. The negative mean drop velocity and positive magnitude of volume flux along the centerline in Fig. 10, shows that the velocity is averaged by particle number and not by particle mass. Also an accurate value of $\mathrm{D}_{30}$ is heavily dependent on measurements of an adequate number of larger drops. For the first two axial locations, except around the centerline, the peak values and the shapes of the flux profiles with and without combustion are very close, indicating negligible drop vaporization and/or burning in our set up. Above $25 \mathrm{~mm}$ the peak of volume flux for the burning case are lower than the non-burning case due to combustion, similar trends were shown by McDonnell and Samuelsen ${ }^{(17)}$. 
For the 45 degrees swirl plate, no difference in volume flux can be seen as is compared with 30 degrees plate. However, the peak of 45 degrees swirl plate shifts slightly outward toward the combustion wall.

For the 30 degrees plate, the results of volume flux were integrated over the spray area to obtain the total liquid fuel flow rate. Plots of the total liquid flow rate with respect to the axial position are shown in Fig. 11 for reacting and nonreacting cases. Results for the reacting case as compared with the total liquid volume flow rate from the nozzle measured by a rotameter of $0.67 \mathrm{cc} / \mathrm{min}$ show $1,78.5,80.6,82.4$, and 96 percent fuel evaporation at axial positions of $25,40,55,70$, and $90 \mathrm{~mm}$ from the nozzle, respectively. This trend is in agreement with Edwards et al. ${ }^{(18)}$. The results for the 45 degrees swirl plate showed similar results. For the nonburning case, it shows the same trend but lower evaporation and more dispersion (also confirmed by visual observation) of the droplets. For this case, the results are not reliable due to incorrect volume flux measurement by PDPA at low and negative velocities around the spray center line.

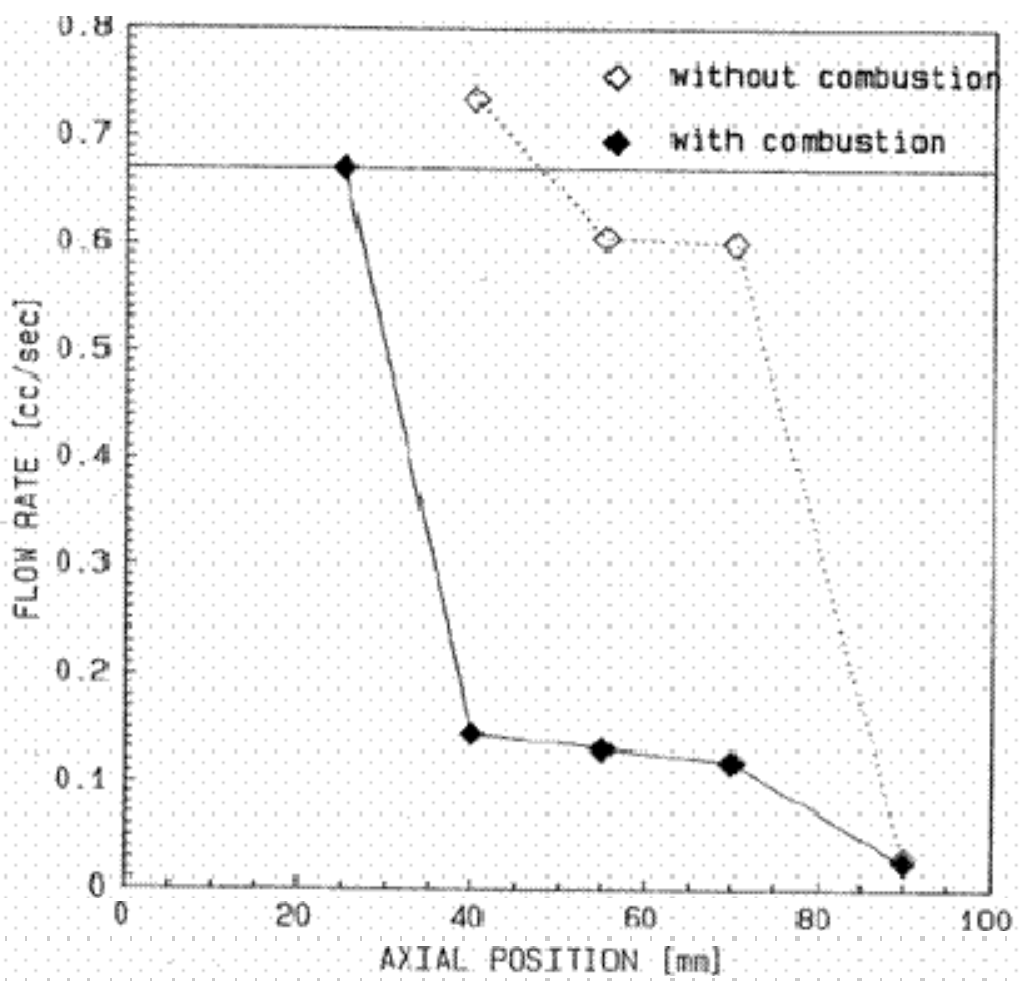

Fig. 11. Liquid volume flow rate as a function of axial position with combustion

Using the volume flux profiles of Figs. 4 and 10 and the photographs of the flame, three radial zones became apparent at most axial positions (see Fig.3). A preheat/reaction zone extends from the outer edge of the spray (beyond which drop velocity measurement is not possible due to absence of drops) radially inward to the outer edge of the visible flame-brush zone. A turbulent flame-brush zone exists from this outer edge of the flame to the peak of the mean temperature profile. Finally, a burned gases zone lies inside the spray flame where toroidal recirculation exists (from the peak temperature to the spray axis).

\section{Summary and Conclusions}

Parametric studies were carried out to optimize the optics parameters such as PM tube voltages and frequency shift. The swirling flow produced by the swirl plate establishes a strong recirculation zone that is essential for flame stabilization under the operating conditions. Recirculating of hot gasses push back towards the center of the chamber has shown to improve fuel utilization, and to promote flame stabilization. The key to stabilize the flame is to eliminate unnecessary turbulence within the chamber. For the 45 degree swirl plate, higher values of RMS drop velocity were observed in the chamber. The 30 degree 
plate lowers the turbulence, which causes a more stable flame.

Very close to the nozzle, a conical liquid sheet with nominal included angle of $30^{\circ}$ (cold region) exists that disintegrates into droplets. Evaporation of fuel begins at an axial position $10 \mathrm{~mm}$. Radial widening of the spray occurs from this location such that a conical-shaped turbulent flame-brush zone is established. It is observed that the radially-shifted axial mean drop velocity profile without combustion and the extrapolated mean axial drop velocity with combustion approach the zero value at the same radial position as very near the line called "nominal spray line". Close to the nozzle, the velocity difference between the droplets with combustion and the radially shifted no combustion case is larger and decreases downstream from the nozzle. This is the manifestation of momentum exchange between drops and gas phase medium. The spray near the nozzle up to axial position of $40 \mathrm{~mm}$ is nonuniform with smaller droplets at the periphery and larger ones at the contour of maximum mean drop velocity. This trend is different at axial position of $40 \mathrm{~mm}$ and $55 \mathrm{~mm}$ with larger droplets near the centerline and smaller ones at the preheat/reaction zone. Above $55 \mathrm{~mm}$, the larger droplets are concentrated at the contour of maximum mean drop velocity with smaller ones at the periphery.

Results for mean axial drop velocity profiles indicate widening of the spray, with slight increase in the magnitudes of the peak drop velocities due to combustion. No measurements inside the hollow-cone spray are possible due to burning of fuel droplets. Root-mean-square (RMS) values of drop velocity fluctuations decrease due to combination of increase in gas kinematics viscosity and elimination of small drops at high temperatures. Sauter mean diameter (SMD) radial profiles at all axial locations increase with combustion due to preferential burning of small drops. Fuel volume flux profiles indicated negligible drop vaporization and/or burning up to a distance of $25 \mathrm{~mm}$ from the nozzle.

\section{References}

(1) Labs J. and Parker T., J. of Atomization and Spray, 13 (1): 45-62 JAN-FEB 2003.

(2) Oh S.H., Kim D.I., and Paek M.S., Atomization and Sprays 11 (6): 775-788 NOV-DEC 2001.

(3) Widmann J.F., Presser C. and Leigh S.D., Measurement Science \& Technology 12 (8): 1180-1190 AUG 2001.

(4) Widmann J.F., Presser C. and Leigh S.D., Identifying burst splitting events in phase Doppler interferometry measurements, ATOMIZATION AND SPRAYS 11 (6): 711-733 NOV-DEC 2001.

(5) Strakey P.A., Talley D.G., Sankar S.V. and Bachalo W.D., Phase-Doppler interferometry with probe-to-droplet size ratios less than unity. II. Application of the technique, APPLIED OPTICS 39 (22): 3887-3893 AUG 2000.

(6) Gupta, A.K., Lilley D.G. and Syred N., Swirl Flow Abacus Press, Cambridge, Massachusetts (1984).

(7) Saumweber C., Friedman J.A., and Renksizbulut M., Particle \& particle systems characterization 14 (5): 233-242 OCT 1997.

(8) Bulzan, D.L., J. of Propulsion and Power. (6): 1093-1102 NOV-DEC 1995.

(9) Gupta A.K., Damm T., Charagundla S.R. and Presser C., 16 (5): 845-852 SEP, 2000.

(10) Bachalo W.D., "The Phase Doppler Method: Analysis and Application", Optical Particle Sizing, pp.283-299, Plenum, 1988.

(11) Bachalo W.D. and Houser, M.J., "Phase Doppler Spray Analyzer for Simultaneous Measurement of Drop Size and Velocity Distribution", Optical Engineering, Vol. 23, No. 4, pp. 583, 1984.

(12) Sankar S.V. and Bachalo WD, "Response Characteristics of the Phase Doppler Particle Analyzerfor Sizing Spherical Particles Larger than the Light Wavelength", Applied Optics, Vol. 30, No. 12, pp. 487-1496, 1991.

(13) Sankar S.V., Weber B.J., Kamemoto D.Y. and Bachalo W.D., "Sizing Fine Particles with thePhase Doppler Interferometric Technique", Applied Optics, Vol. 30, No. 33, pp. 4914-4920, 1991.

(14) Zhu J.Y., Rudolf R.C., Bachalo E.J. and Bachalo W.D., "Number Density and Mass Flux Measurements Using the Phase Doppler Particle Analyzer in Reacting and Non9. Reacting Swirling Flows", A1AA 93-0361, 1993.

(15) Bachalo W.D., Rudoff R.C. and Brena de la Rosa A., AIAA-88-02636. 26th Aerospace 
Science Meeting, Reno, Nevada, 1988.

(16) Chehroudi, B., "Preliminary Drop Size and Velocity Measurements in a Dense Diesel-type Spray, SAE International off-Highway \& Powerplants Congress \& Exposition, SAE Paper 901673, 1990, Milwaukee, Wisconsin.

(17) McDonell V.G. and Samuelsen G.S., 2nd Volume, ASTM STP 1083, American Society for Testing and Materials, Philadelphia, pp. 170-189 (1990).

(18) Edwards C.F., Rudoff R.C. and Bachalo W.D., Fifth International Symposium on the Applications of Laser Technique to Fluid Mechanics, Lisbon, Portugal, 1990. 